\title{
Poor Sleep and Emotion Dysregulation Mediate the Association between Depressive and Premenstrual Symptoms in Young Adult Women: Supplemental File
}

In order to assess the impact of imputed values on the associations between depressive symptoms, sleep quality, dysregulated emotions and pre-menstrual symptoms, analyses were rerun including only participants who had no missing data $(n=248)$. Table S1 shows demographic information for included participants. Table S2 displays correlation coefficients for pre-menstrual symptoms, depressive symptoms, sleep quality and emotion dysregulation. Figure $\mathrm{S} 1$ shows the primary mediation analyses with separate models assessing sleep and emotion dysregulation as mediators of the association between depressive and pre-menstrual symptoms. Finally, Figures S2a-c replicate the exploratory analyses conducted in the main paper.

In order to account for the co-measurement of sleep disruption and depression different across scales, scale totals were also calculated without the inclusion of items addressing sleep disturbance (MDQ and CESD) and depression (MDQ). Table S3 shows the revised descriptive statistics and correlation coefficients for each scale. Figure S3 shows the primary mediation analyses conducted using scales with items removed, and Figures S4a-c replicate the exploratory analyses found in the main paper. 
Table S1. Demographic information for participants with no missing values $(n=248)$.

\begin{tabular}{|c|c|c|}
\hline & Mean (SD) / \% (n) & Median (IQR) \\
\hline Age & $20.73(1.80)$ & $20.00(3.00)$ \\
\hline \multicolumn{3}{|l|}{ Race } \\
\hline White & $35.1(87)$ & $\mathrm{N} / \mathrm{A}$ \\
\hline African-American & $13.3(33)$ & $\mathrm{N} / \mathrm{A}$ \\
\hline Asian & $29.0(72)$ & $\mathrm{N} / \mathrm{A}$ \\
\hline Mixed Race & $5.6(14)$ & $\mathrm{N} / \mathrm{A}$ \\
\hline Other & $8.1(20)$ & $\mathrm{N} / \mathrm{A}$ \\
\hline Prefer not to specify & $6.9(17)$ & $\mathrm{N} / \mathrm{A}$ \\
\hline \multicolumn{3}{|l|}{ Ethnicity } \\
\hline Non-Hispanic & $50.8(126)$ & $\mathrm{N} / \mathrm{A}$ \\
\hline Hispanic & $41.9(104)$ & $\mathrm{N} / \mathrm{A}$ \\
\hline Mixed & $2.0(5)$ & $\mathrm{N} / \mathrm{A}$ \\
\hline Other & $2.4(6)$ & $\mathrm{N} / \mathrm{A}$ \\
\hline Prefer not to specify & $2.8(7)$ & $\mathrm{N} / \mathrm{A}$ \\
\hline \multicolumn{3}{|l|}{ Menstrual Cycle Regularity } \\
\hline Usually or always irregular & $18.1(45)$ & $\mathrm{N} / \mathrm{A}$ \\
\hline Somewhat regular & $46.4(115)$ & $\mathrm{N} / \mathrm{A}$ \\
\hline Always regular & $35.1(87)$ & $\mathrm{N} / \mathrm{A}$ \\
\hline Unknown/Prefer not to answer & $0.4(1)$ & $\mathrm{N} / \mathrm{A}$ \\
\hline \multicolumn{3}{|c|}{ Moos Menstrual Distress Questionnaire } \\
\hline Total & $62.33(20.19)$ & $58.00(22.00)$ \\
\hline Pain & $13.55(4.06)$ & $13.50(7.00)$ \\
\hline Concentration & $11.94(4.99)$ & $10.00(6.00)$ \\
\hline Behavioral Change & $10.29(3.74)$ & $10.00(6.00)$ \\
\hline Automatic Reactions & $5.65(2.45)$ & $5.00(2.00)$ \\
\hline Water Retention & $6.13(2.33)$ & $5.00(3.00)$ \\
\hline
\end{tabular}




\begin{tabular}{lll}
\hline Negative Affect & $15.73(5.89)$ & $15.00(9.00)$ \\
Arousal & $8.49(3.54)$ & $7.00(6.00)$ \\
Control & $7.54(3.16)$ & $6.00(1.00)$ \\
Center for Epidemiologic Studies Depression Scale Total & $17.49(11.24)$ & $16.00(16.00)$ \\
(clinical cut off: 16 or above) & & \\
Difficulties in Emotion Regulation Scale Total (range 36-180) & $83.77(23.31)$ & $83.50(35.00)$ \\
Pittsburgh Sleep Quality Index Total (scores >5 indicate poor & $6.34(3.00)$ & $6.00(4.00)$ \\
sleep quality) & & \\
\hline
\end{tabular}


Table S2: Correlation coefficients between study variables $(n=428)$.

\begin{tabular}{llll}
\hline & CESD & PSQI & DERS \\
\hline MDQ & $.56^{*}$ & $.44^{*}$ & $.53^{*}$ \\
CESD & & $.45^{*}$ & $.75^{\star}$ \\
PSQI & & & $.35^{\star}$
\end{tabular}

MDQ: Menstrual Distress Questionnaire; CESD: Center for Epidemiologic Studies Depression Scale;

PSQI: Pittsburgh Sleep Quality Index; DERS: Difficulty in Emotion Regulation Scale. ${ }^{*} p<.001$. 
Figure S1. Direct and indirect pathways for primary mediation analyses for participants with no missing data $(n=248)$.

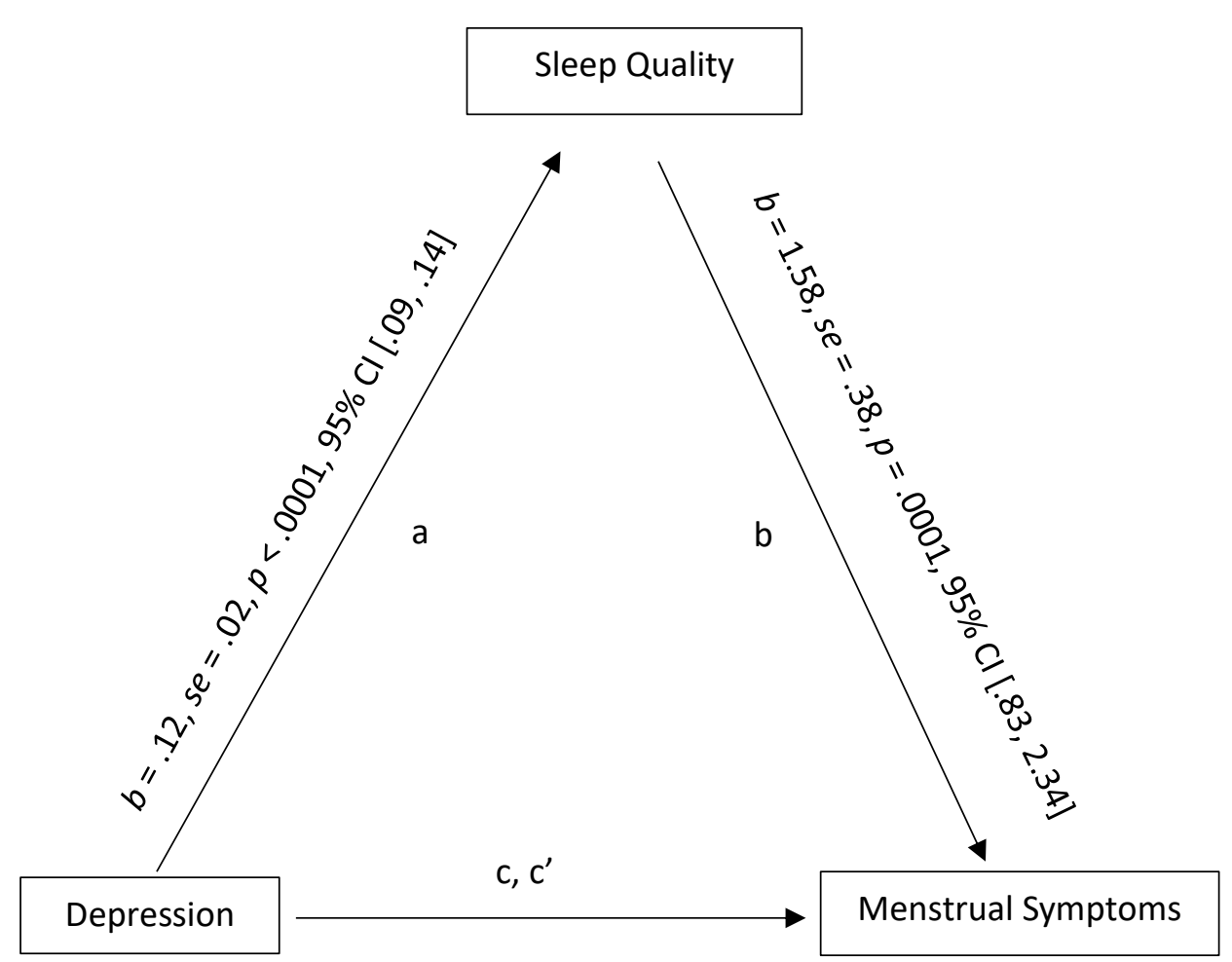

Direct Effect (c): $b=1.01, s e=.09, p<.0001,95 \% \mathrm{Cl}[.82,1.20]$

Mediated Direct Effect $\left(c^{\prime}\right): b=.82$, se $=.10, p<.0001,95 \% \mathrm{Cl}[.62,1.02]$

Indirect Effect (ab): $b=.19$, se $=.06,95 \% \mathrm{Cl}[.07, .32]$

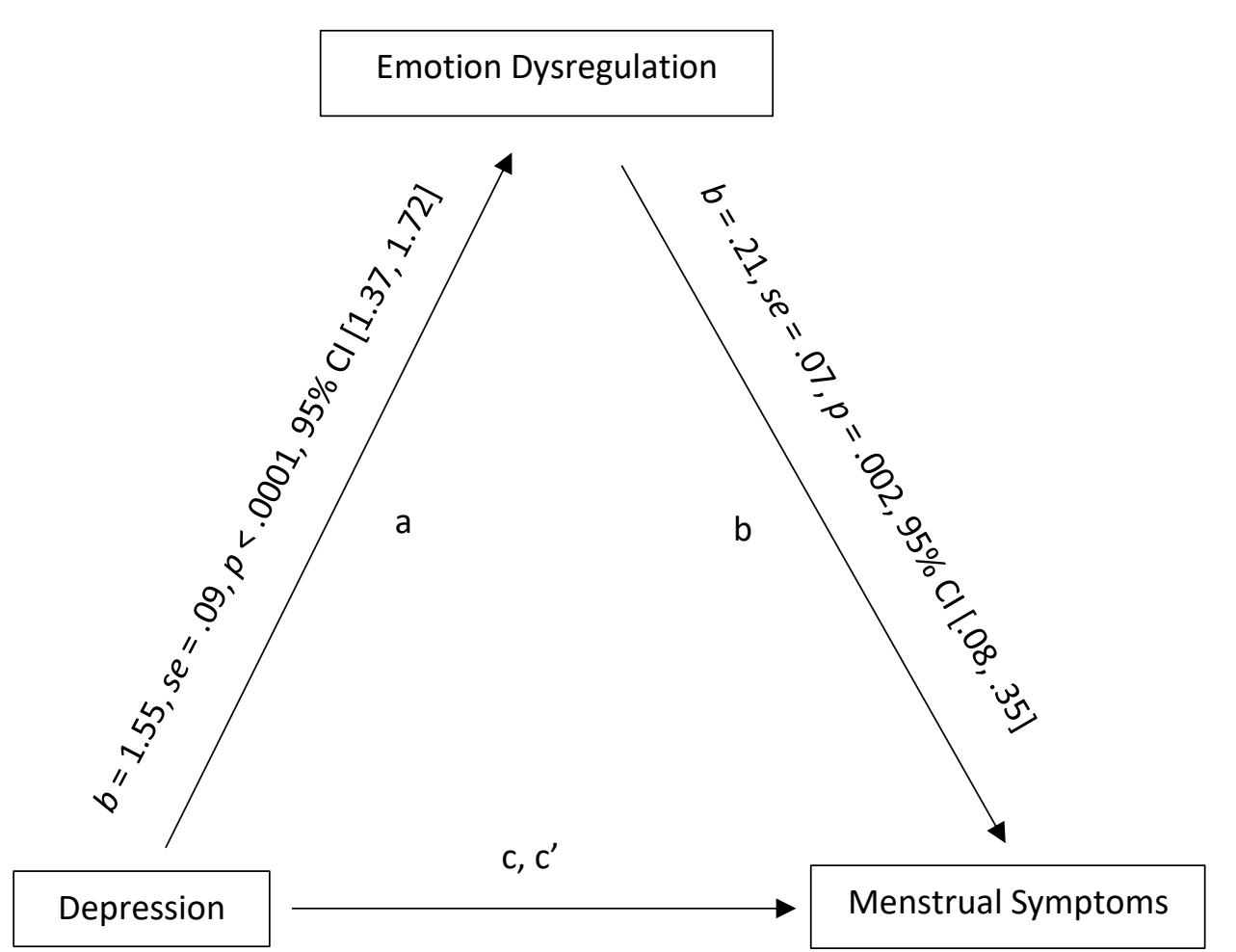

Direct Effect (c): $b=1.01, s e=.09, p<.0001,95 \% \mathrm{Cl}[.82,1.20]$

Mediated Direct Effect $\left(\mathrm{c}^{\prime}\right): b=.67, \mathrm{se}=.14, p<.0001,95 \% \mathrm{Cl}[.40, .95]$ Indirect Effect (ab): $b=.33$, se $=.13,95 \% \mathrm{Cl}[.08, .60]$ 
Figure S2a: Direct and indirect pathways for exploratory mediation analyses $(n=248)$ : Pre-Menstrual Pain.

Dashed line indicates non-significant pathway.

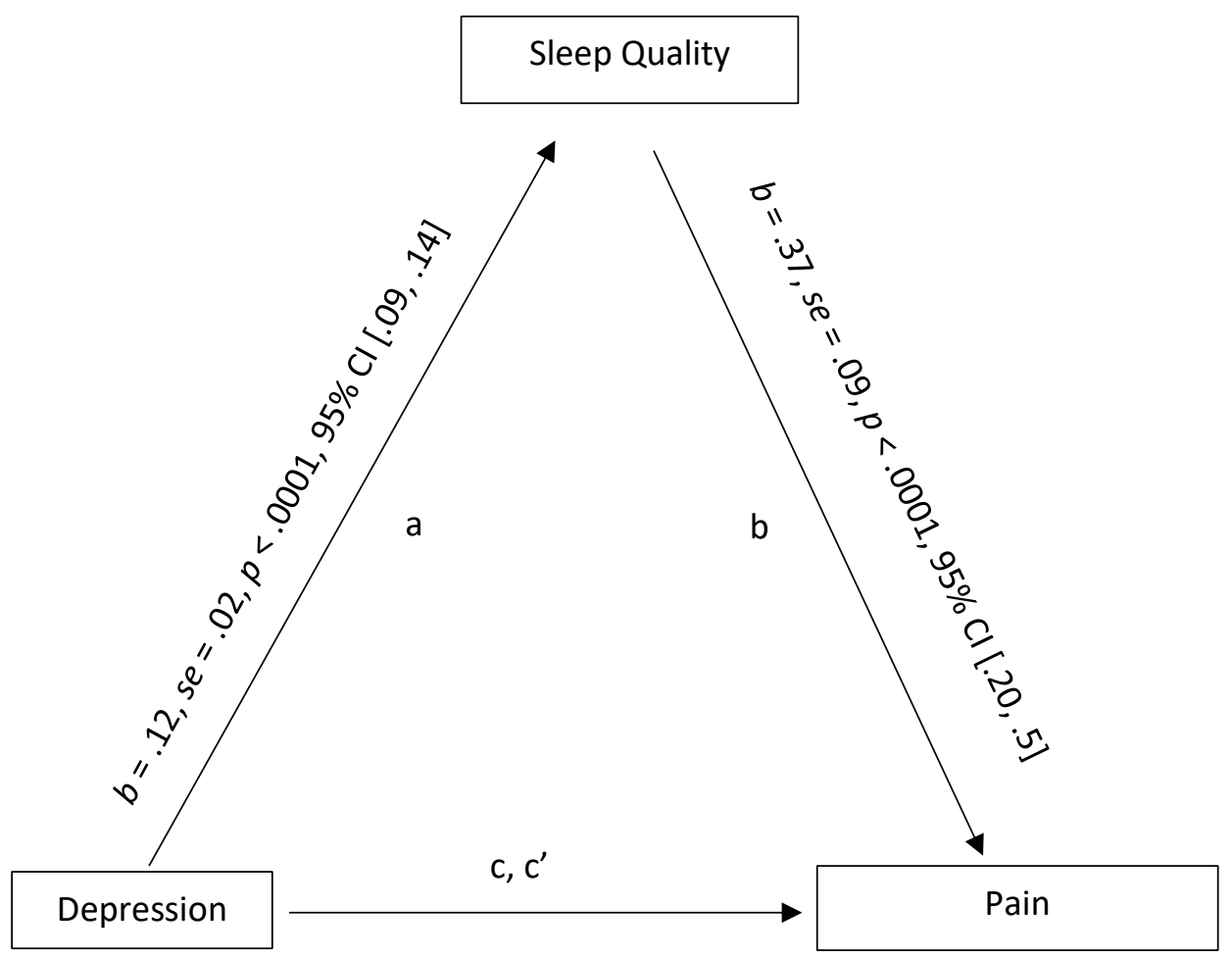

Direct Effect (c): $b=.12, \mathrm{se}=.02, p<.0001,95 \% \mathrm{Cl}[.07, .16]$

Mediated Direct Effect $\left(\mathrm{c}^{\prime}\right): b=.07$, se $=.02, p=.002,95 \% \mathrm{Cl}[.03, .12]$

Indirect Effect (ab): $b=.04$, se $=.01,95 \% \mathrm{Cl}[.02, .07]$

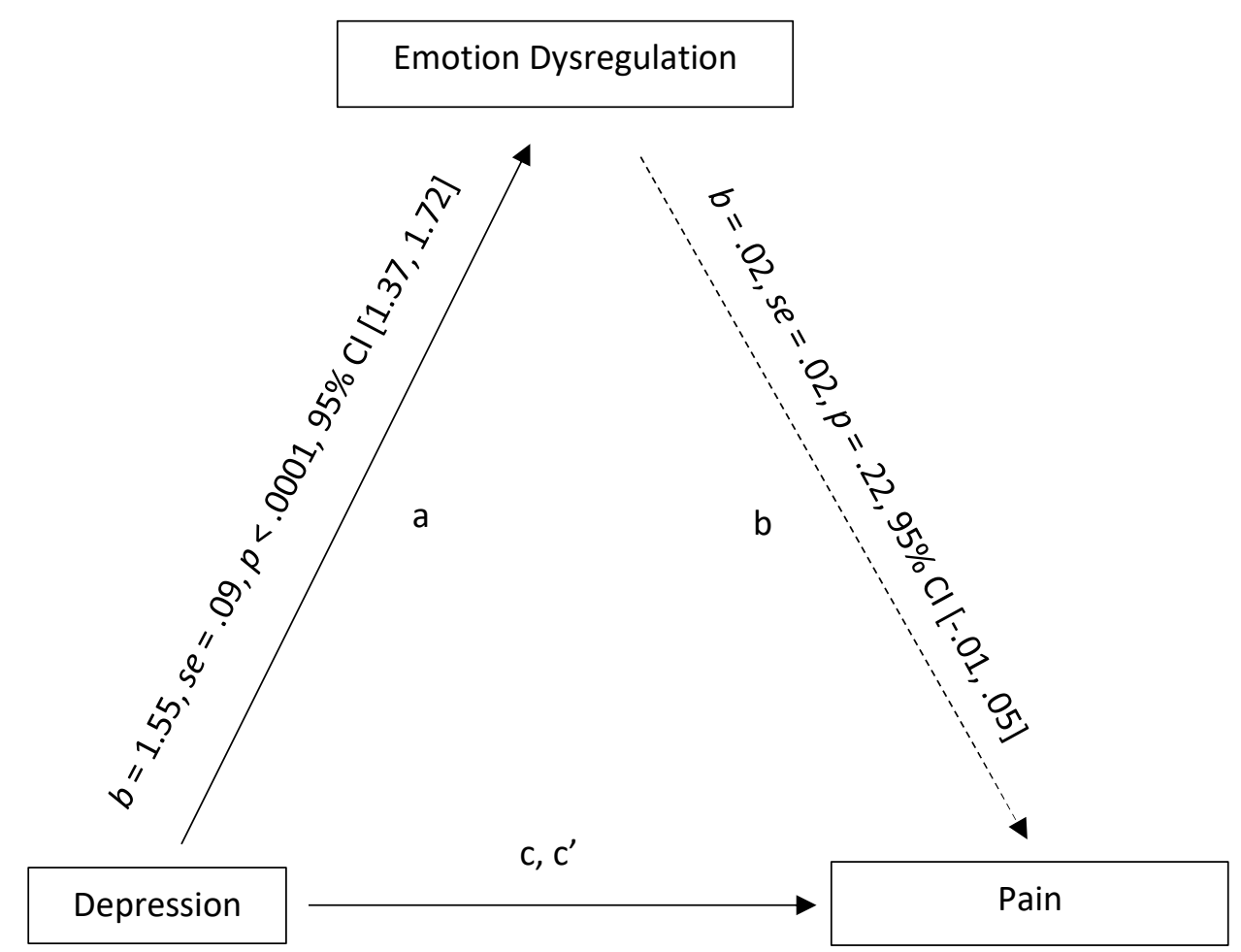

Direct Effect (c): $b=.12$, se $=.02, p<.0001,95 \% \mathrm{Cl}[.07, .16]$

Mediated Direct Effect $\left(c^{\prime}\right): b=.08, s e=.03, p=.009,95 \% \mathrm{Cl}[.02, .15]$

Indirect Effect (ab): $b=.03$, se $=.03,95 \% \mathrm{Cl}[-.02, .08]$ 
Figure S2b: Direct and indirect pathways for exploratory mediation analyses $(n=248)$ : Pre-Menstrual Concentration.

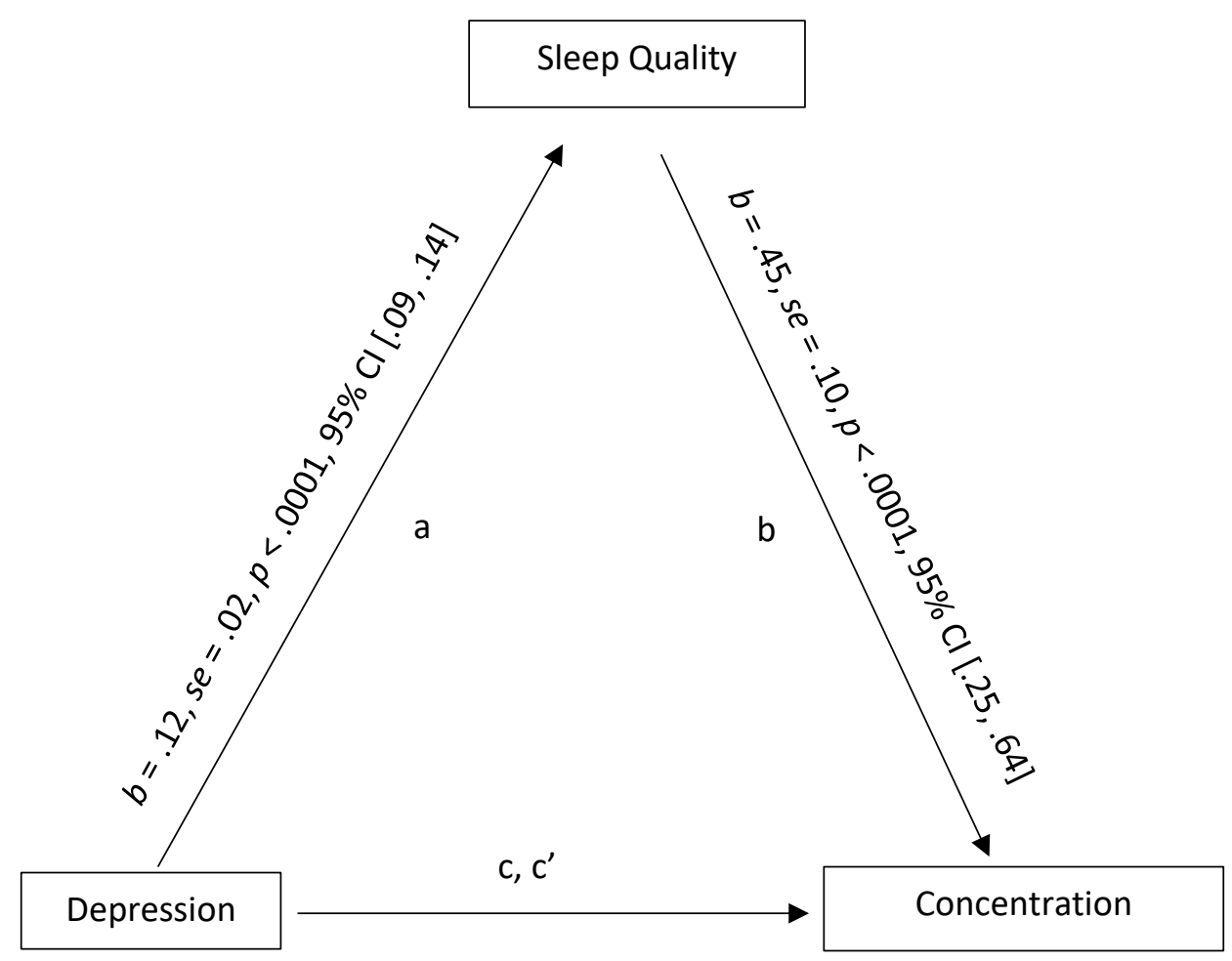

Direct Effect (c): $b=.22, s e=.02, p<.0001,95 \% \mathrm{Cl}[.17, .27]$

Mediated Direct Effect $\left(c^{\prime}\right): b=.17, s e=.02, p<.0001,95 \% \mathrm{Cl}[.12, .22]$

Indirect Effect (ab): $b=.05$, se $=.02,95 \% \mathrm{Cl}[.02, .09]$

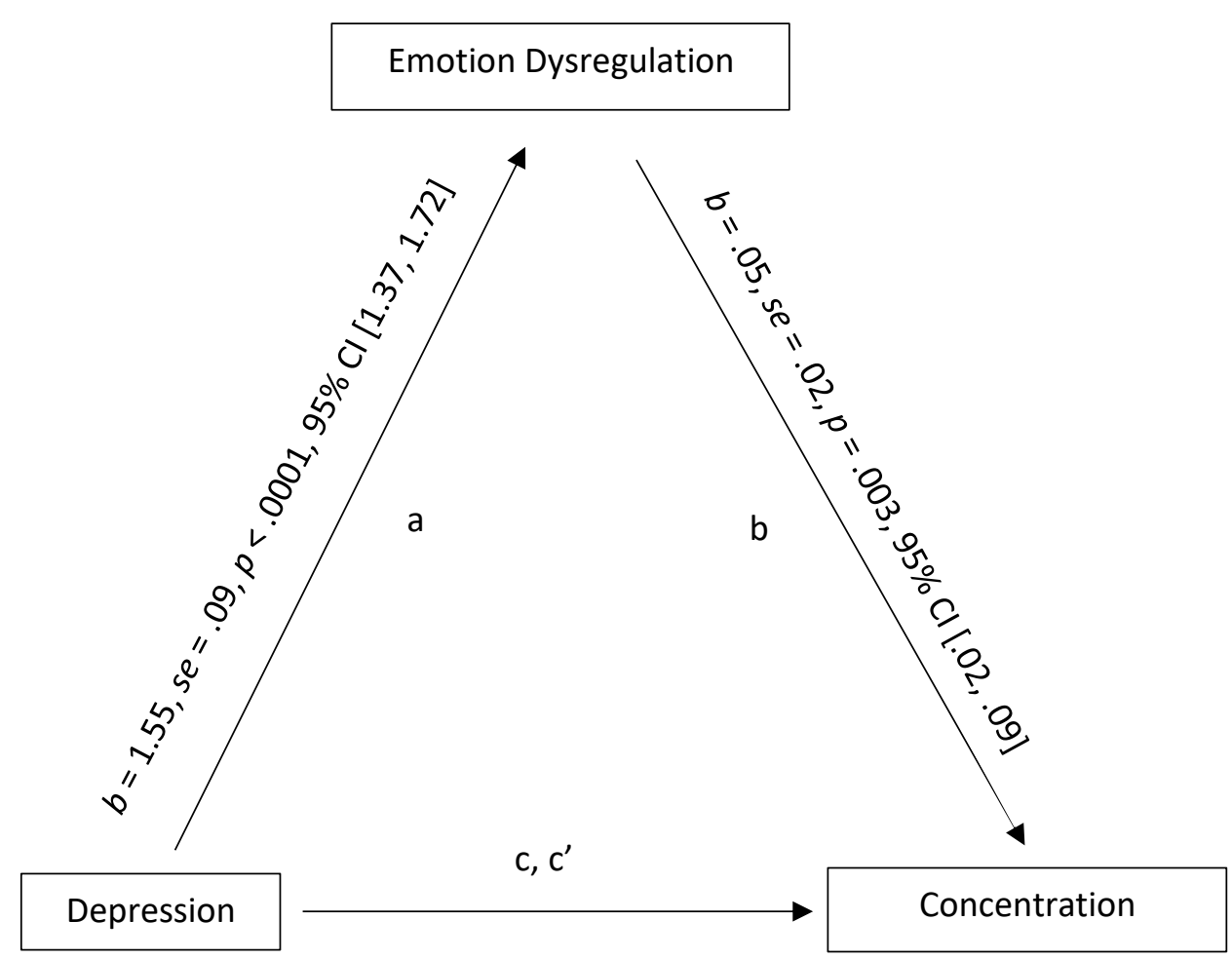

Direct Effect (c): $b=.22$, se $=.02, p<.0001,95 \% \mathrm{Cl}[.17, .27]$

Mediated Direct Effect (c'): $b=.14$, se $=.04, p=.0002,95 \% \mathrm{Cl}[.07, .21]$

Indirect Effect $(\mathrm{ab}): b=.08$, se $=.03,95 \% \mathrm{Cl}[.02, .15]$ 
Figure S2c: Direct and indirect pathways for exploratory mediation analyses $(n=248)$ : Pre-Menstrual Negative Affect.

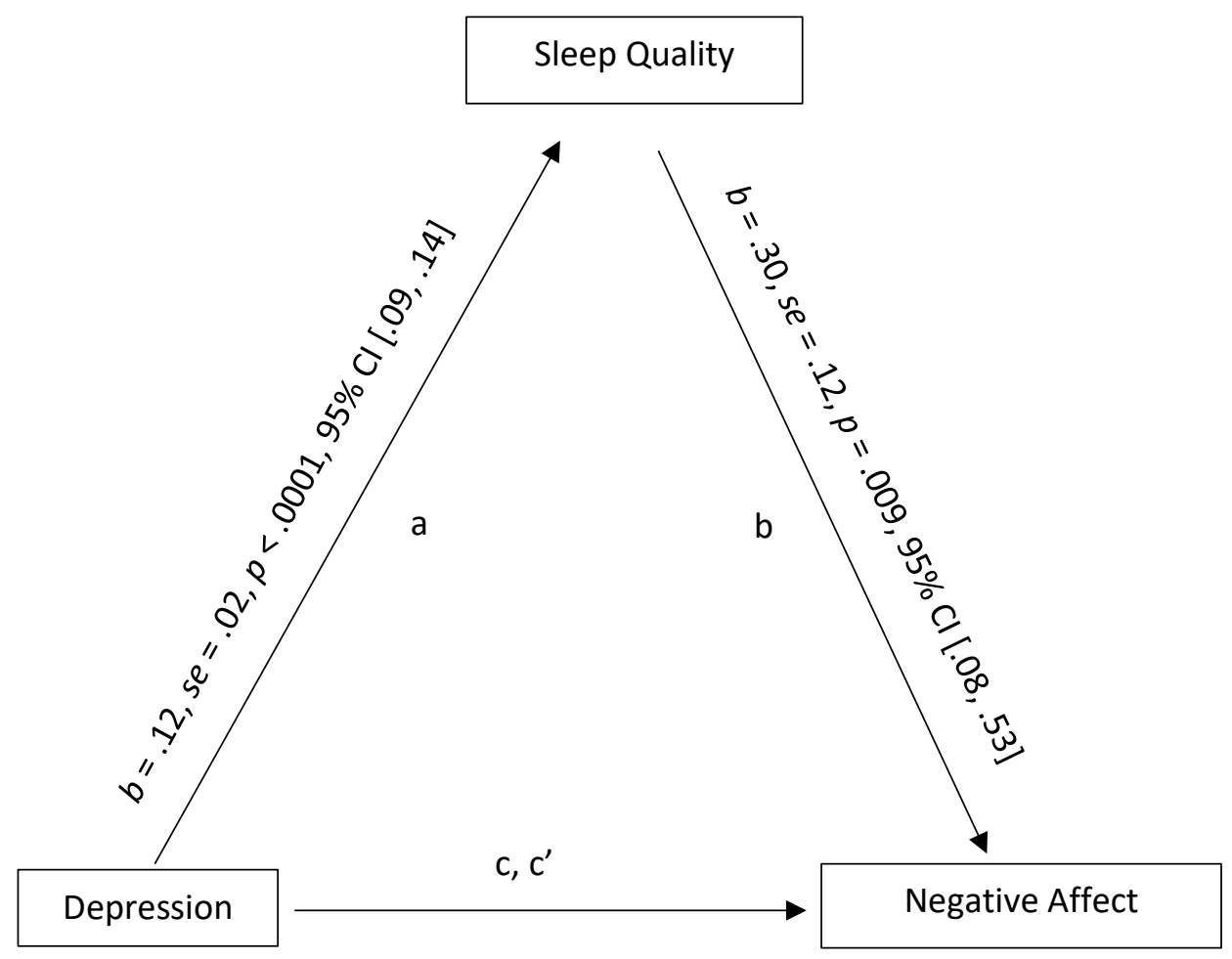

Direct Effect (c): $b=.29, s e=.03, p<.0001,95 \% \mathrm{Cl}[.23, .34]$

Mediated Direct Effect $\left(c^{\prime}\right): b=.25$, se $=.03, p<.0001,95 \% \mathrm{Cl}[.19, .31]$

Indirect Effect (ab): $b=.04$, se $=.02,95 \% \mathrm{Cl}[.01, .07]$

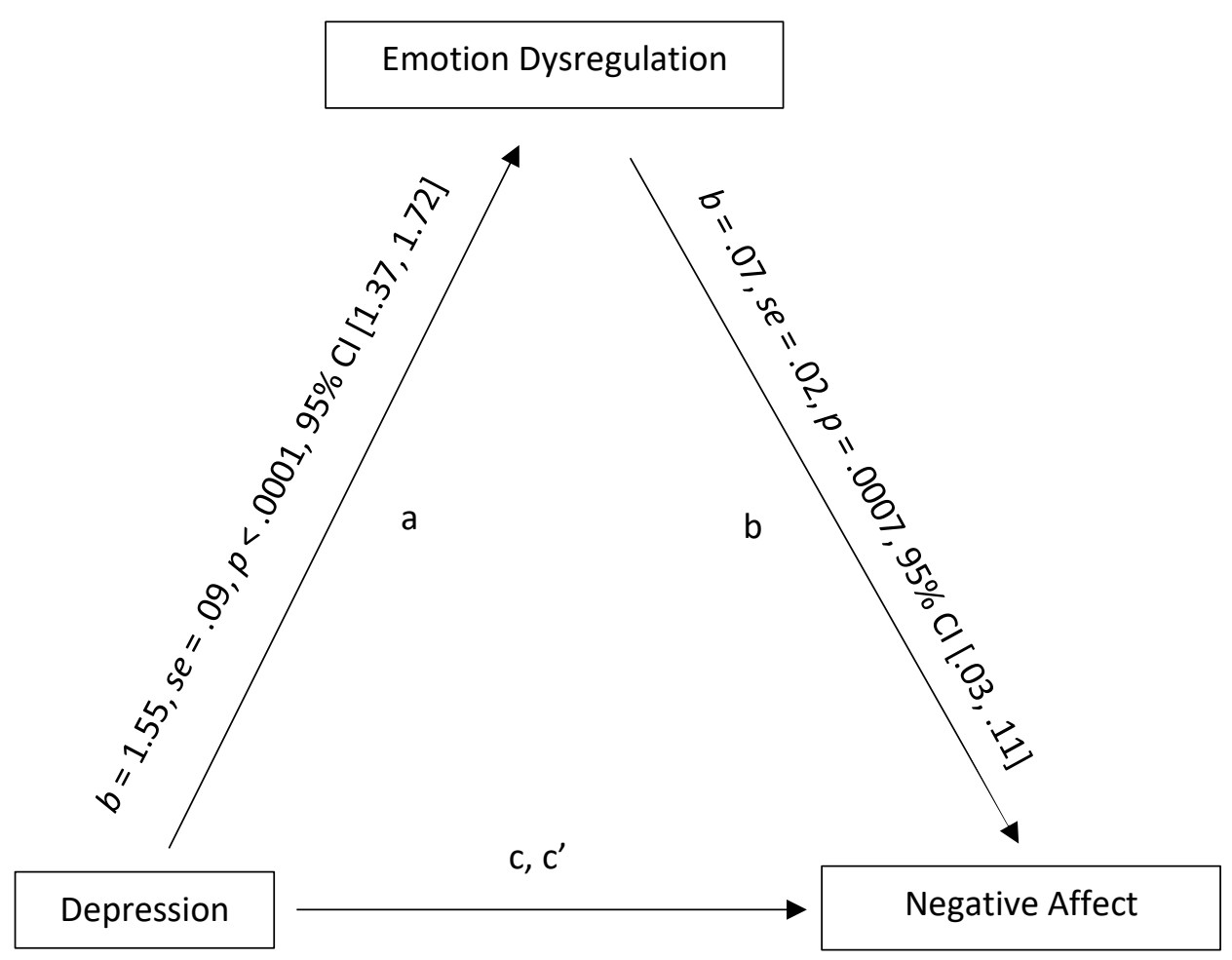

Direct Effect (c): $b=.29, s e=.03, p<.0001,95 \% \mathrm{Cl}[.23, .34]$

Mediated Direct Effect $\left(c^{\prime}\right): b=.18$, se $=.04, p<.0001,95 \% \mathrm{Cl}[.10, .26]$

Indirect Effect $(\mathrm{ab}): b=.11$, se $=.04,95 \% \mathrm{Cl}[.04, .17]$ 
Table S3: Descriptive values and correlation coefficients for scales with overlapping items removed $(n=265)$.

\begin{tabular}{llllll}
\hline & M (SD) & Median (IQR) & CESD & PSQI & DERS \\
\hline MDQ Total (no sleep or & $57.97(18.95)$ & $54.00(22.00)$ & $.55^{*}$ & $.45^{*}$ & $.53^{*}$ \\
depression items) & & & & \\
CESD (no sleep item) & $16.75(10.65)$ & $16.00(16.00)$ & $.44^{*}$ & $.74^{*}$ \\
PSQI & & & & \\
& $6.36(3.05)$ & $6.00(4.00)$ & & $.34^{*}$ \\
DERS & & & & \\
& $83.93(23.35)$ & $84.00(35.00)$ & & \\
\end{tabular}

MDQ: Menstrual Distress Questionnaire; CESD: Center for Epidemiologic Studies Depression Scale; PSQI: Pittsburgh Sleep Quality Index; DERS: Difficulty in Emotion Regulation Scale. ${ }^{*} p<.001$. 
Figure S3. Direct and indirect pathways for primary mediation analyses, overlapping scale items removed $(n=265)$.

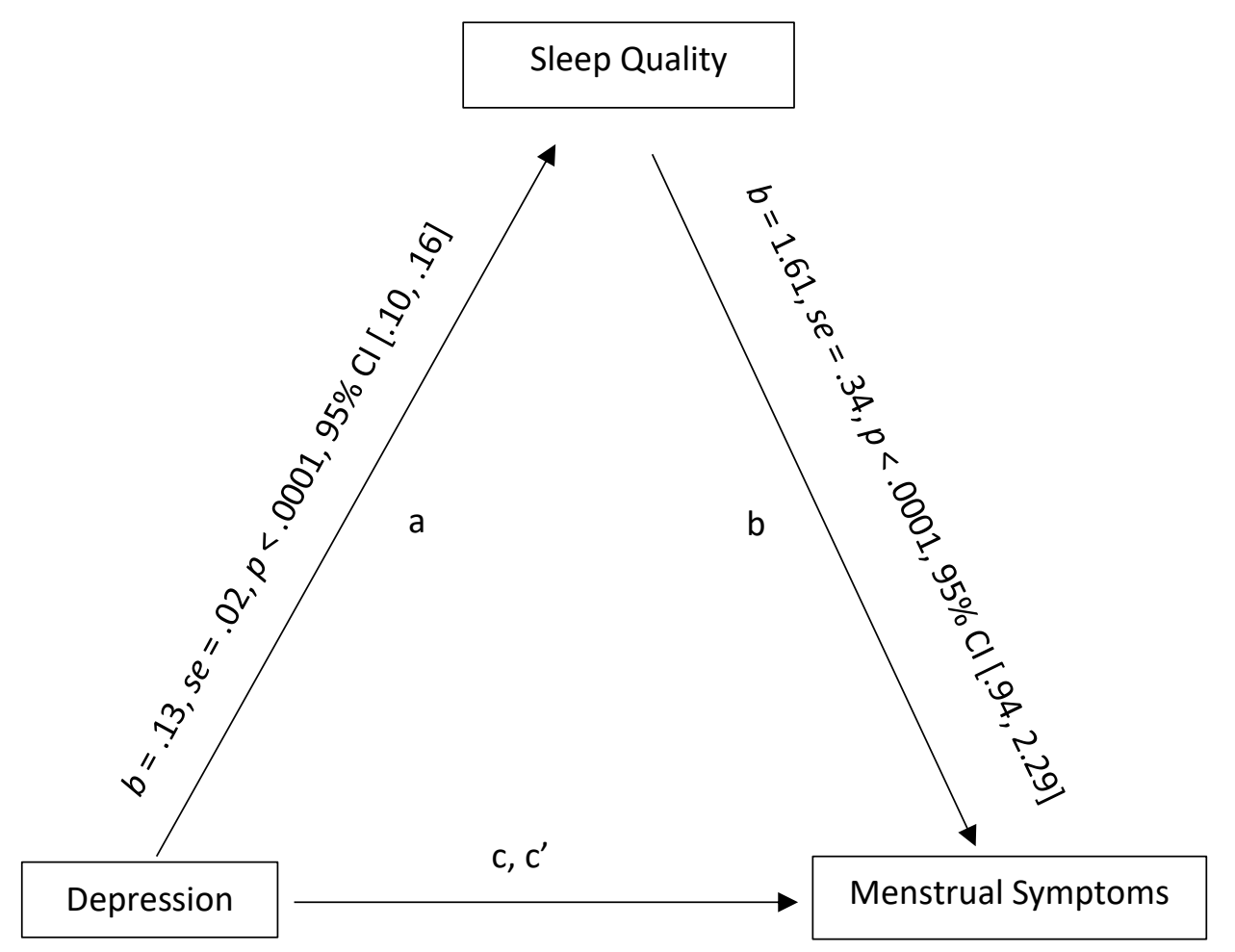

Direct Effect (c): $b=.98, s e=.09, p<.0001,95 \% \mathrm{Cl}[.80,1.16]$

Mediated Direct Effect $\left(c^{\prime}\right): b=.78$, se $=.10, p<.0001,95 \% \mathrm{Cl}[.58, .97]$

Indirect Effect $(\mathrm{ab}): b=.20 . s e=.06,95 \% \mathrm{Cl}[.09, .33]$

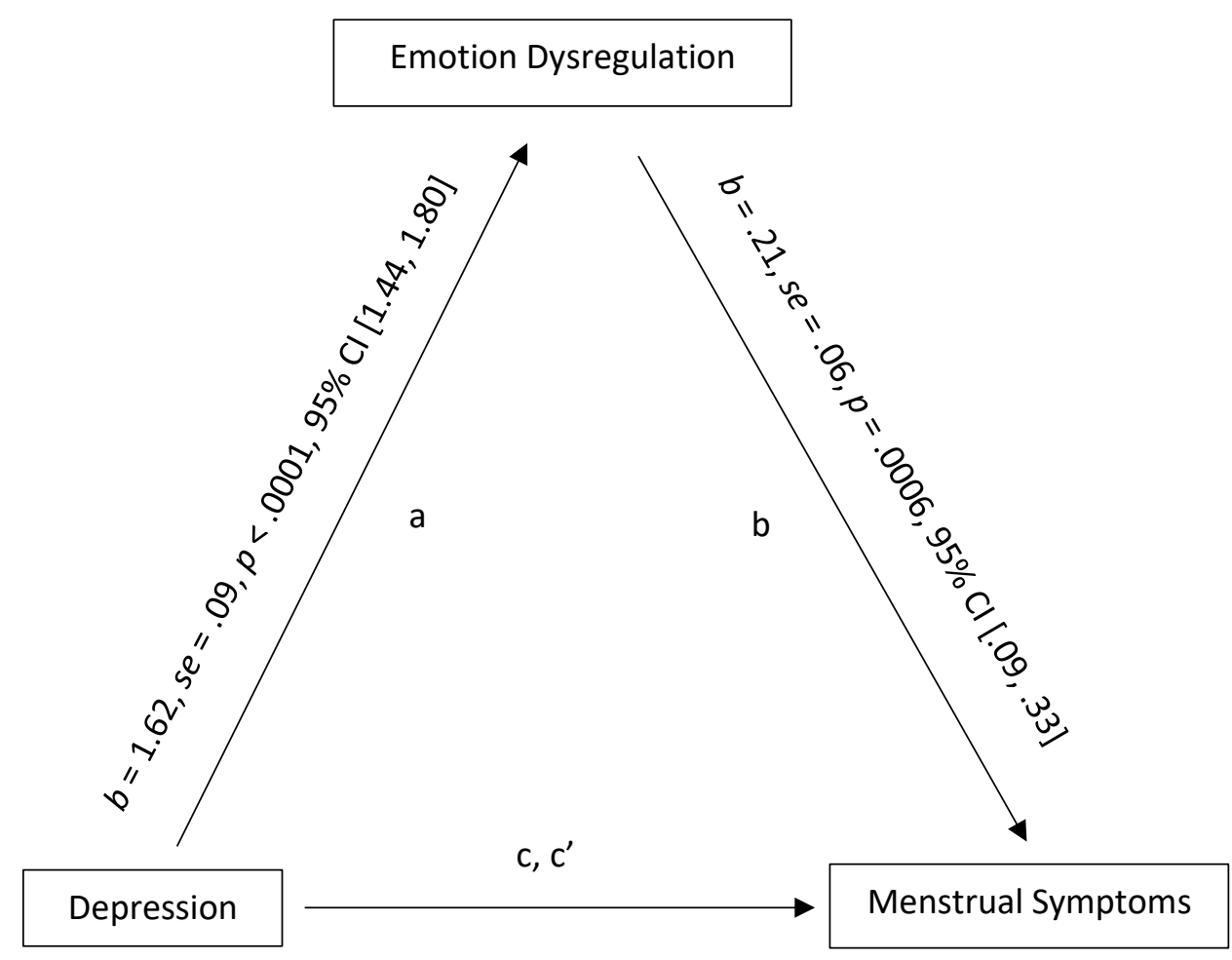

Direct Effect (c): $b=.98, s e=.09, p<.0001,95 \% \mathrm{Cl}[.80,1.16]$ Mediated Direct Effect $\left(c^{\prime}\right): b=.64$, se $=.13, p<.0001,95 \% \mathrm{Cl}[.38, .90]$ Indirect Effect $(\mathrm{ab}): b=.34$, se $=.11,95 \% \mathrm{Cl}[.11, .58]$ 
Figure S4a: Direct and indirect pathways for exploratory mediation analyses with overlapping items removed $(n=265)$ : Pre-Menstrual Pain.

Dashed line indicates non-significant pathway.

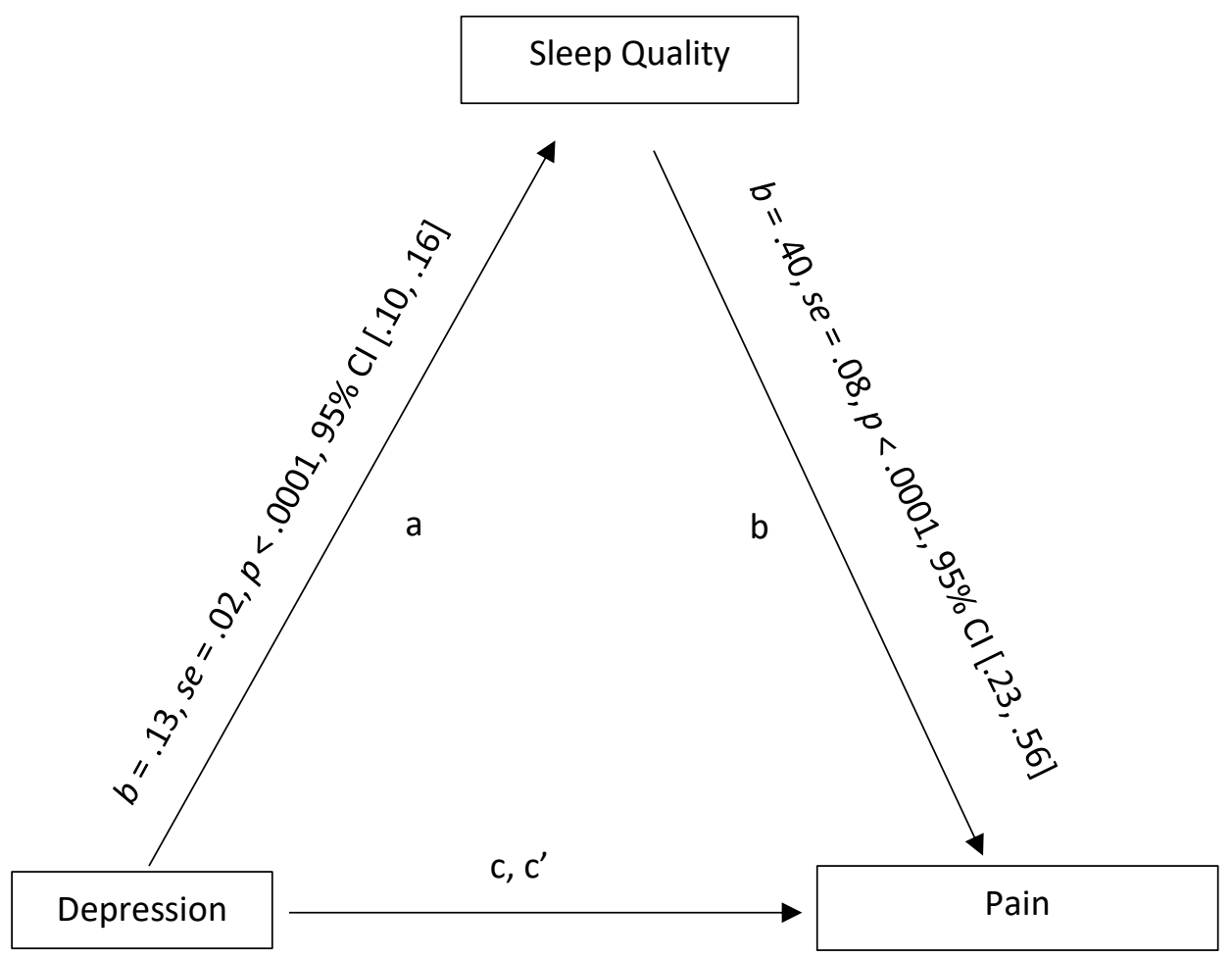

Direct Effect (c): $b=.12$, se $=.02, p<.0001,95 \% \mathrm{Cl}[.08, .17]$ Mediated Direct Effect $\left(\mathrm{c}^{\prime}\right): b=.08$, se $=.02, p=.002,95 \% \mathrm{Cl}[.03, .12]$ Indirect Effect $(\mathrm{ab}): b=.05 . s e=.01,95 \% \mathrm{Cl}[.03, .08]$

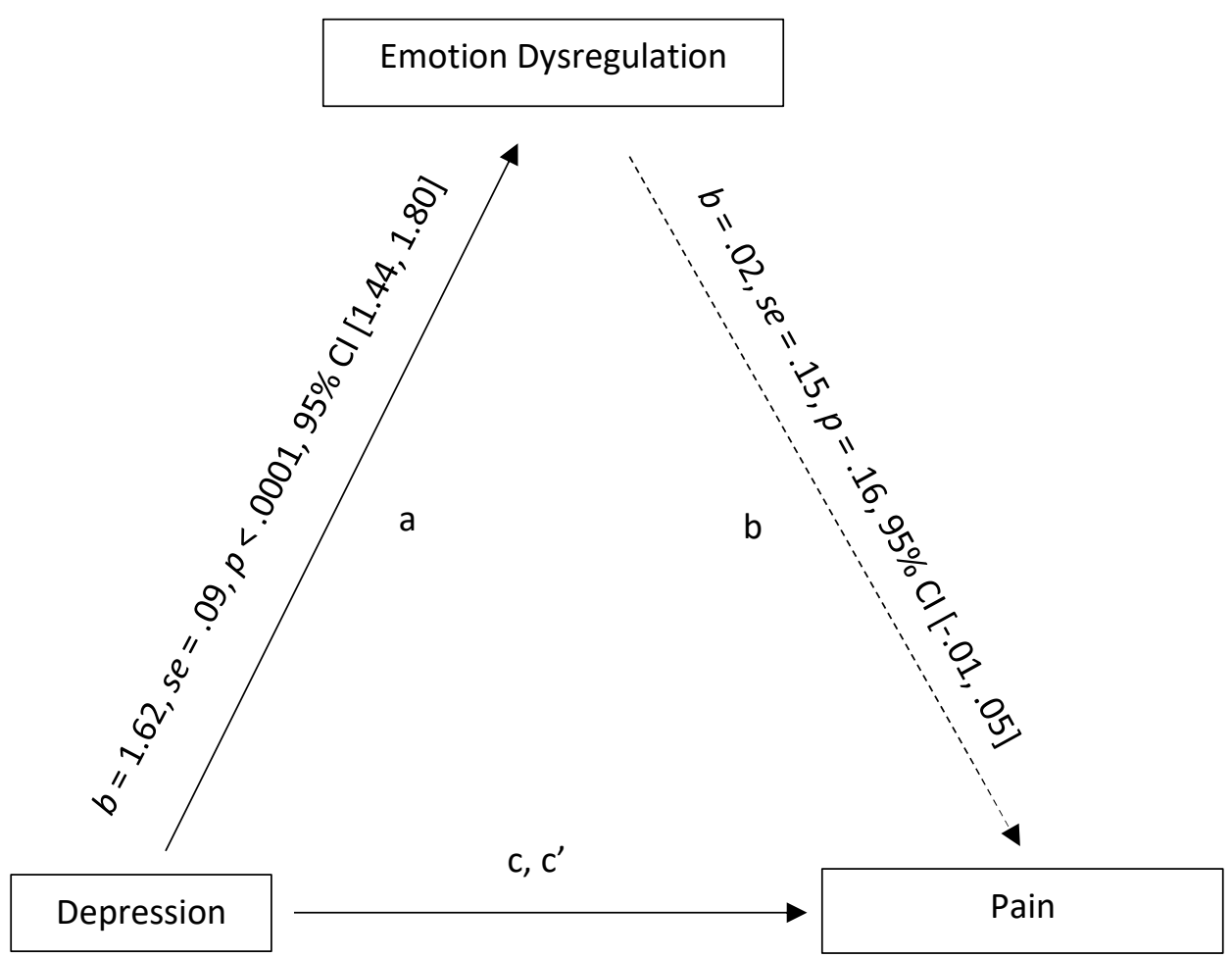

Direct Effect (c): $b=.12, s e=.02, p<.0001,95 \% \mathrm{Cl}[.08, .17]$ Mediated Direct Effect $\left(c^{\prime}\right): b=.09$, se $=.03, p=.007,95 \% \mathrm{Cl}[.03, .16]$ Indirect Effect $(\mathrm{ab}): b=.03, \mathrm{se}=.03,95 \% \mathrm{Cl}[-.02, .08]$ 
Figure S4b: Direct and indirect pathways for exploratory mediation analyses with overlapping items removed $(n=265)$ : Pre-Menstrual Concentration.

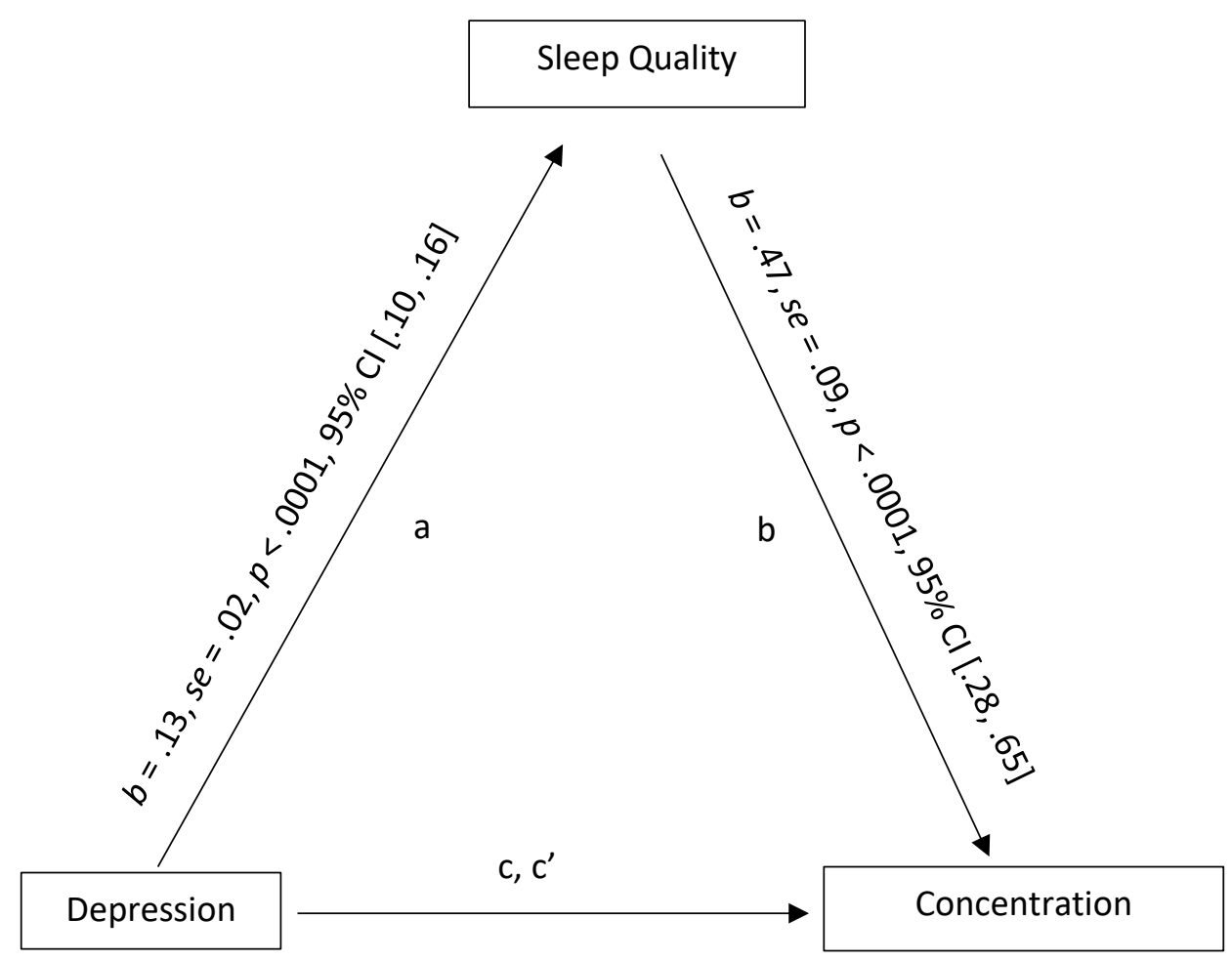

Direct Effect (c): $b=.23, s e=.03, p<.0001,95 \% \mathrm{Cl}[.18, .28]$

Mediated Direct Effect $\left(c^{\prime}\right): b=.17$, se $=.03, p<.0001,95 \% \mathrm{Cl}[.12, .23]$

Indirect Effect (ab): $b=.06 . s e=.02,95 \% \mathrm{Cl}[.03, .10]$

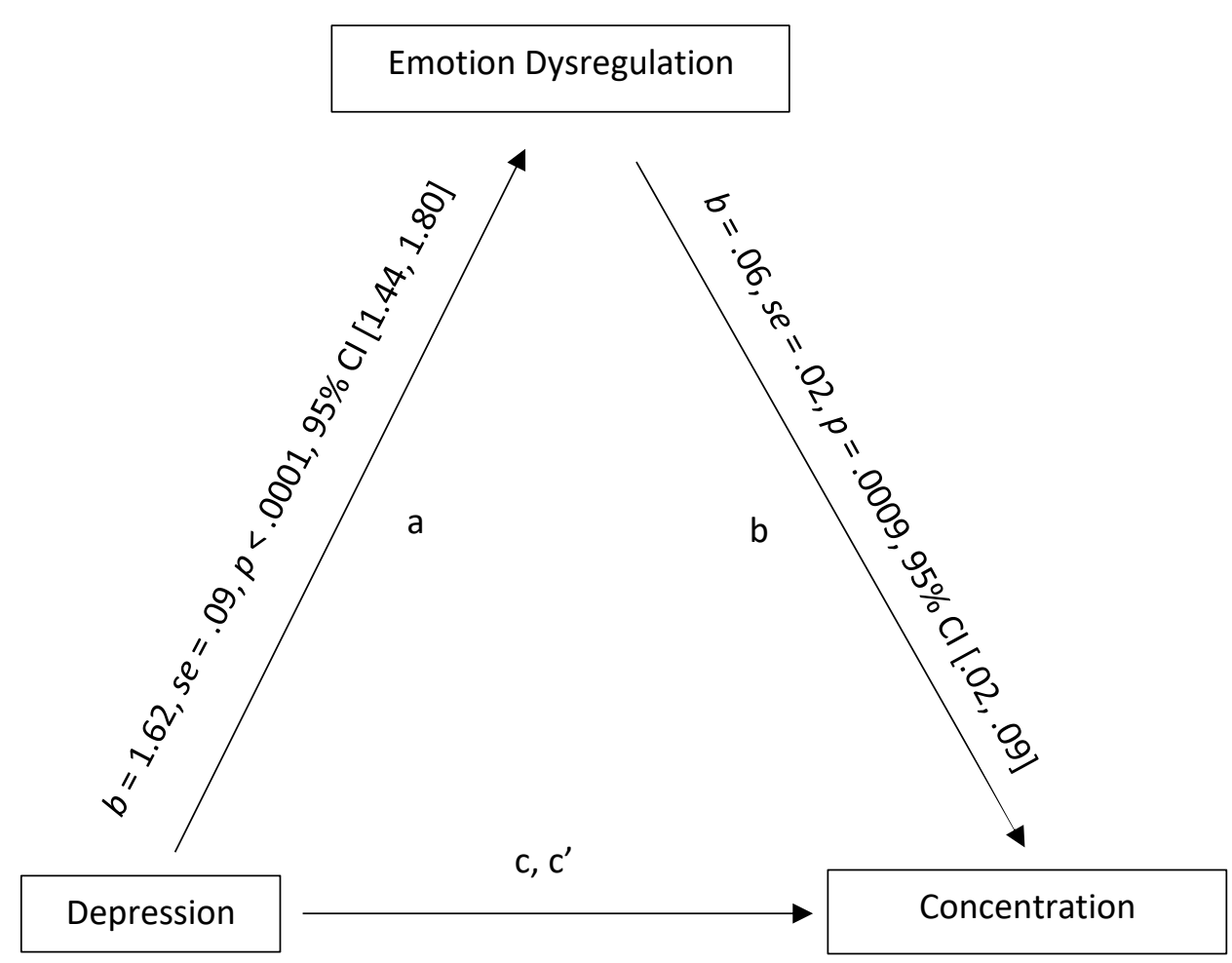

Direct Effect (c): $b=.23, s e=.03, p<.0001,95 \% \mathrm{Cl}[.18, .28]$

Mediated Direct Effect $\left(c^{\prime}\right): b=.14$, se $=.03, p=.0001,95 \% \mathrm{Cl}[.07, .21]$

Indirect Effect $(\mathrm{ab}): b=.09$, se $=.03,95 \% \mathrm{Cl}[.03, .16]$ 
Figure S4c: Direct and indirect pathways for exploratory mediation analyses with overlapping items removed ( $n=265)$ : Pre-Menstrual Negative Affect.

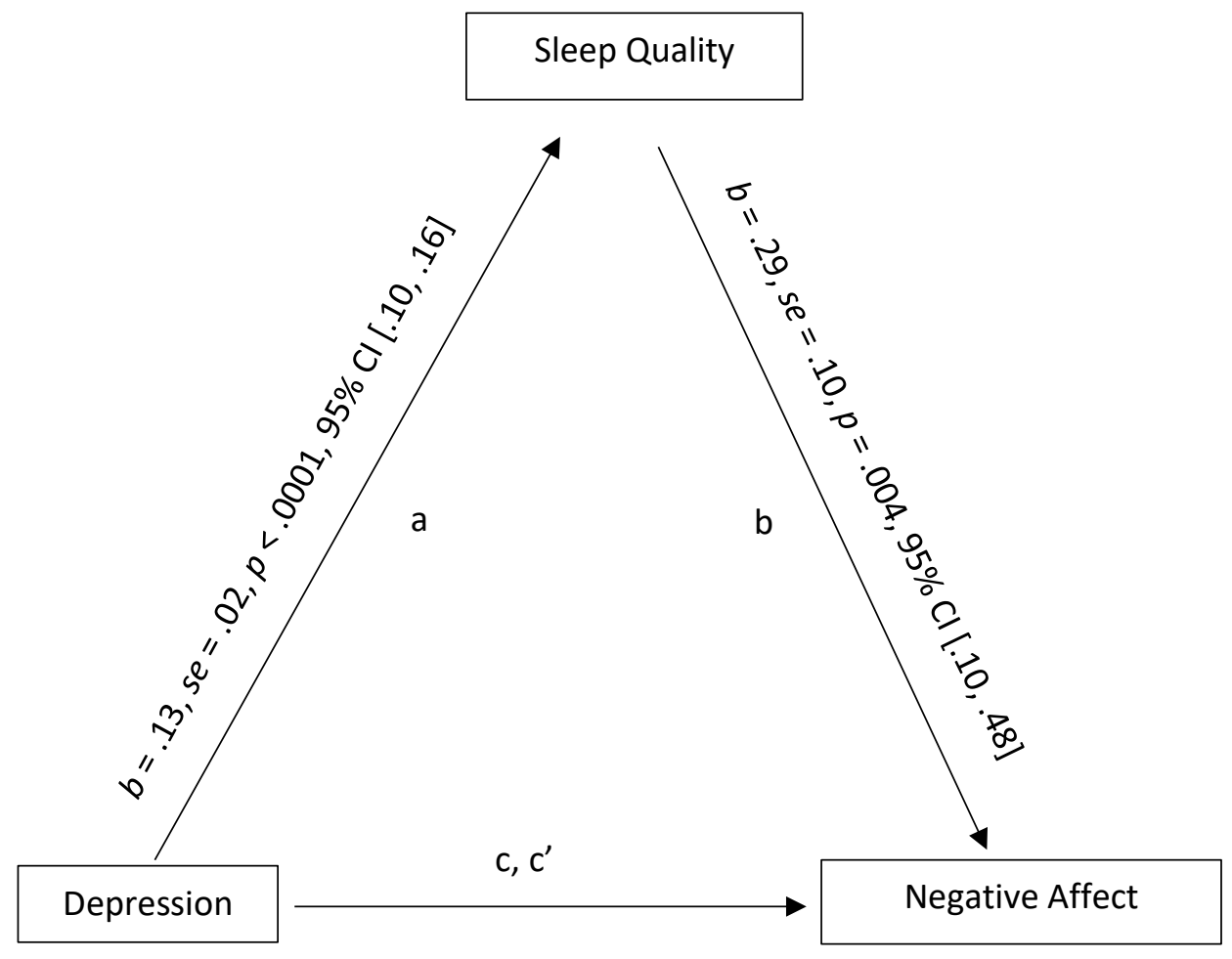

Direct Effect (c): $b=.24, s e=.03, p<.0001,95 \% \mathrm{Cl}[.19, .29]$

Mediated Direct Effect $\left(c^{\prime}\right): b=.20$, se $=.03, p<.0001,95 \% \mathrm{Cl}[.15, .26]$

Indirect Effect (ab): $b=.04$. se $=.01,95 \% \mathrm{Cl}[.01, .07]$

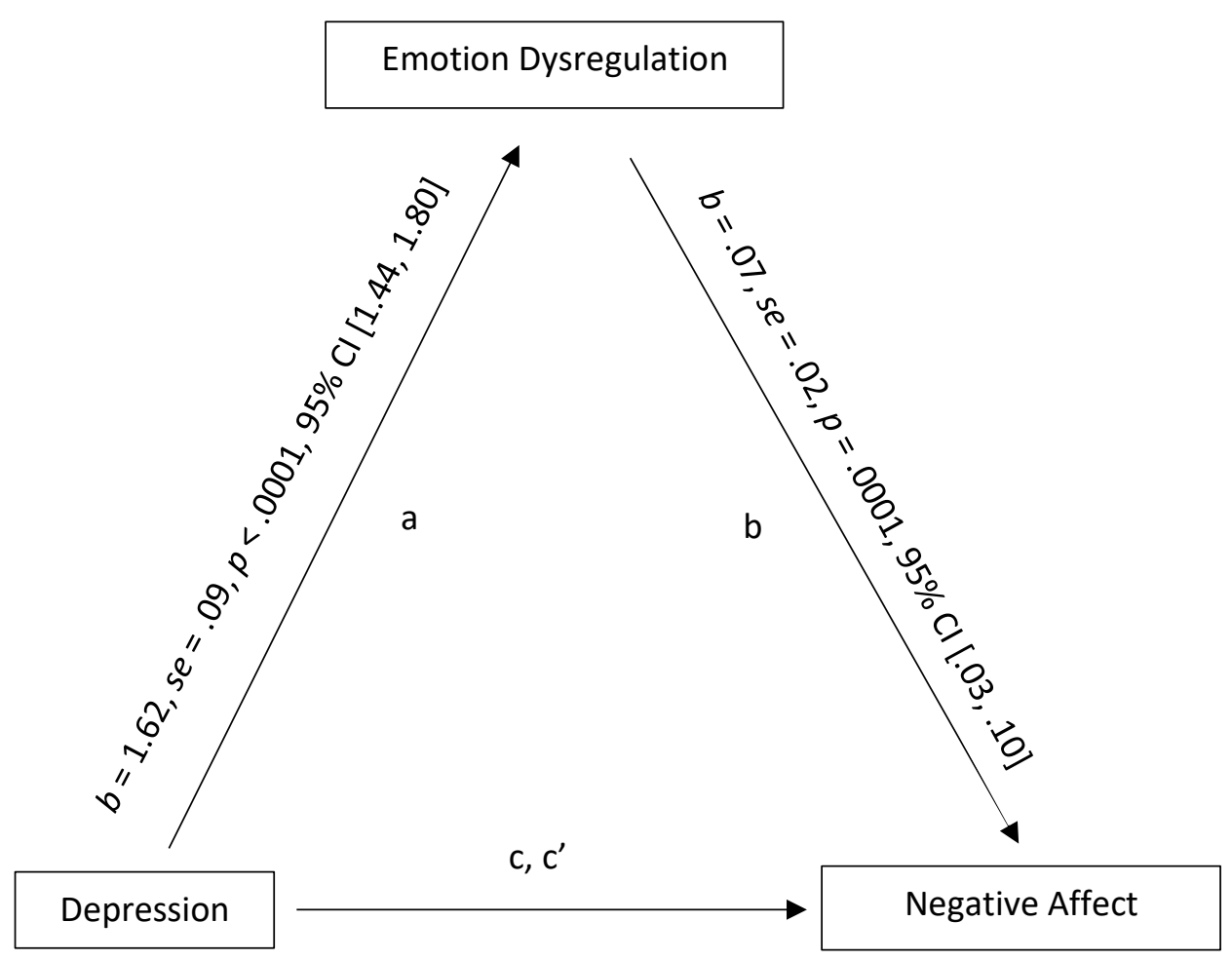

Direct Effect (c): $b=.24, s e=.03, p<.0001,95 \% \mathrm{Cl}[.19, .29]$

Mediated Direct Effect $\left(c^{\prime}\right): b=.13$, se $=.04, p=.0005,95 \% \mathrm{Cl}[.06, .21]$

Indirect Effect $(\mathrm{ab}): b=.11$, se $=.03,95 \% \mathrm{Cl}[.05, .17]$ 